Discrete Comput Geom 33:423-443 (2005)

DOI: $10.1007 / \mathrm{s} 00454-004-1112-8$

\title{
Topology of Definable Hausdorff Limits
}

\author{
Thierry Zell \\ School of Mathematics, Georgia Institute of Technology, \\ Atlanta, GA 30332-0160, USA \\ zell@math.gatech.edu
}

\begin{abstract}
Let $A \subseteq \mathbb{R}^{n+r}$ be a set definable in an o-minimal expansion $\mathcal{S}$ of the real field, let $A^{\prime} \subseteq \mathbb{R}^{r}$ be its projection, and assume that the non-empty fibers $A_{a} \subseteq \mathbb{R}^{n}$ are compact for all $a \in A^{\prime}$ and uniformly bounded, i.e. all fibers are contained in a ball of fixed radius $B(0, R)$. If $L$ is the Hausdorff limit of a sequence of fibers $A_{a_{i}}$, we give an upper-bound for the Betti numbers $b_{k}(L)$ in terms of definable sets explicitly constructed from a fiber $A_{a}$. In particular, this allows us to establish effective complexity bounds in the semialgebraic case and in the Pfaffian case. In the Pfaffian setting, Gabrielov introduced the relative closure to construct the o-minimal structure $\mathcal{S}_{\text {Pfaff }}$ generated by Pfaffian functions in a way that is adapted to complexity problems. Our results can be used to estimate the Betti numbers of a relative closure $(X, Y)_{0}$ in the special case where $Y=\varnothing$.
\end{abstract}

\section{Introduction}

We consider a bounded subset $A \subseteq \mathbb{R}^{n+r}$ which is definable in an o-minimal expansion $\mathcal{S}$ of the real field (the reader can refer to [7] or [8] for definitions). Let $A^{\prime}$ be the canonical projection of $A$ in $\mathbb{R}^{r}$, and for all $a \in A^{\prime}$ we define the fiber $A_{a}$ as $A_{a}=\left\{\mathbf{x} \in \mathbb{R}^{n} \mid\right.$ $(\mathbf{x}, a) \in A\}$. Assume that these fibers are compact for all $a \in A^{\prime}$. Note that since we assumed that $A$ was bounded, the fibers $A_{a}$ are all contained in a ball $B(0, R)$ for some $R>0$. Recall that for compact subsets $A$ and $B$ of $\mathbb{R}^{n}$, we can define the Hausdorff distance between $A$ and $B$ as

$$
d_{\mathrm{H}}(A, B)=\max _{\mathbf{x} \in A} \min _{\mathbf{y} \in B}|\mathbf{x}-\mathbf{y}|+\max _{\mathbf{y} \in B} \min _{\mathbf{x} \in A}|\mathbf{x}-\mathbf{y}|
$$

The Hausdorff distance gives the space $\mathcal{K}_{n}$ of compact subsets of $\mathbb{R}^{n}$ a metric space structure.

If $\left(a_{i}\right)$ is a sequence in $A^{\prime}$, and $L$ is a compact subset of $\mathbb{R}^{n}$ such that the limit of the sequence $d_{\mathrm{H}}\left(A_{a_{i}}, L\right)$ is zero, we call $L$ the Hausdorff limit of the sequence $A_{a_{i}}$. It 
is a well-established fact that when $A$ is definable in an o-minimal structure $\mathcal{S}$, then the Hausdorff limit $L$ is also definable in $\mathcal{S}$ : it was first proved by Bröcker [4] in the algebraic case; in the general case, it follows from the definability of types that was first proved by Marker and Steinhorn [22], and later by Pillay [24]. Recently, direct proofs were suggested, one using model theoretic arguments by van den Dries [9] and a purely geometric one by Lion and Speissegger [21].

\section{Main Result}

In this paper we investigate how the topology of the Hausdorff limit can be related to the topology of the fibers $A_{a}$ and their Cartesian powers. To do so, we need to introduce for any integer $p$ a distance function $\rho_{p}$ on $(p+1)$-tuples $\left(\mathbf{x}_{0}, \ldots, \mathbf{x}_{p}\right)$ of points in $\mathbb{R}^{n}$ by

$$
\rho_{p}\left(\mathbf{x}_{0}, \ldots, \mathbf{x}_{p}\right)=\sum_{0 \leq i<j \leq p}\left|\mathbf{x}_{i}-\mathbf{x}_{j}\right|^{2}
$$

(where $|\mathbf{x}|$ is the Euclidean distance in $\mathbb{R}^{n}$ ). The expanded $p$ th diagonal of $A_{a}$ is defined for all $\delta>0$ by $D_{a}^{0}(\delta)=A_{a}$ and for $p \geq 1$,

$$
D_{a}^{p}(\delta)=\left\{\left(\mathbf{x}_{0}, \ldots, \mathbf{x}_{p}\right) \in\left(A_{a}\right)^{p+1} \mid \rho_{p}\left(\mathbf{x}_{0}, \ldots, \mathbf{x}_{p}\right) \leq \delta\right\} .
$$

Let $b_{k}(L)$ denote the $k$ th Betti number of $L$, by which we mean the rank of the singular homology group $H_{k}(L, \mathbb{Z})$. Our main result is the following upper-bound.

Theorem 1. Let $A \subseteq \mathbb{R}^{n+r}$ be a bounded definable set with compact fibers and let $L$ be the Hausdorff limit of some sequence $A_{a_{i}}$. Then there exists $a \in A^{\prime}$ and $\delta>0$ such that for any integer $k$ we have

$$
b_{k}(L) \leq \sum_{p+q=k} b_{q}\left(D_{a}^{p}(\delta)\right)
$$

where the set $D_{a}^{p}(\delta)$ is the expanded pth diagonal defined in (2).

The proof of this theorem relies on the construction of a continuous surjection from some fiber $A_{a}$ to $L$, and the use of the spectral sequence associated to such a surjection that was already used in [15]. The spectral sequence alone does not provide directly an estimate in terms of the topology of explicit sets such as the sets $D_{a}^{p}(\delta)$ : the bound (3) is finally obtained after an approximation process.

Thus, Theorem 1 allows us to estimate the Betti numbers of $L-$ which is a definable set, but not obviously so-in terms of the Betti numbers of the sets $D_{a}^{p}(\delta)$ which are not only clearly definable, but also easy to describe from a formula defining $A$. In particular, if $A$ is defined by a quantifier-free formula, the sets $D_{a}^{p}(\delta)$ can also be described without quantifiers. In the semialgebraic setting, this allows us to give good effective bounds on the Betti numbers of Hausdorff limits, since the terms $b_{q}\left(D_{a}^{p}(\delta)\right)$ are easy to bound. When $A$ is given by a quantifier-free formula (see Definition 29), we obtain the following estimates. 
Corollary 2. Let $A \subseteq \mathbb{R}^{n+r}$ be a bounded semialgebraic set with compact fibers and defined by a quantifier-free sign condition $\Phi(\mathbf{x}, a)$ on a family

$$
\mathcal{P}=\left\{p_{1}(\mathbf{x}, a), \ldots, p_{s}(\mathbf{x}, a)\right\}
$$

of polynomials (where $\mathbf{x} \in \mathbb{R}^{n}$ and $a \in \mathbb{R}^{r}$ ). Let $L$ be the Hausdorff limit of some sequence of fibers $A_{a_{i}}$. If $\operatorname{deg}_{\mathbf{x}}\left(p_{j}\right) \leq d$ for all $1 \leq j \leq s$, we have for any integer $k$,

$$
b_{k}(L) \leq O\left(k^{2} s^{2} d\right)^{(k+1) n} .
$$

In particular, the Betti numbers of the Hausdorff limit do not depend on the degrees in $a$ of the polynomials of $\mathcal{P}$.

\section{Application to the Pfaffian Structure}

The present work was motivated by the case where $\mathcal{S}$ is the o-minimal structure generated by Pfaffian functions. This class of real-analytic functions was introduced by Khovanskî̌ [18]; it contains many of the so-called tame functions that can appear in applications, such as real elementary functions or Liouville functions. They are also the basis for the theory of fewnomials, the study of the behavior of real polynomials in terms of the number of monomials that appear with a non-zero coefficient. (See Section 4 for definitions.) Wilkie proved in [26] that Pfaffian functions generate an o-minimal structure $\mathcal{S}_{\text {Pfaff }}$; this result was generalized in [17], [20], and [25].

Pfaffian functions are endowed with a natural notion of complexity, or format (see Definition 25), which is a tuple of integers that control their behavior. This translates easily into a notion of format for sets defined by quantifier-free formulas (called semiPfaffian sets). However, the structure $\mathcal{S}_{\text {Pfaff }}$ contains sets that cannot be defined by a quantifier-free sign condition on Pfaffian functions. In [11] Gabrielov gave an alternative to Wilkie's construction of $\mathcal{S}_{\text {Pfaff }}$, showing that definable sets could be constructed by allowing the operation of relative closure on one-parameter couples of semi-Pfaffian sets. The object of this construction was to extend the notion of format to all Pfaffian sets, and use it to generalize the quantitative results already known for semi- and sub-Pfaffian sets (see the survey [13] and references).

The relative closure is defined as follows: we consider $X$ and $Y$ semi-Pfaffian subsets of $\mathbb{R}^{n} \times \mathbb{R}_{+}$as families of semi-Pfaffian subsets of $\mathbb{R}^{n}$ depending on a parameter $\lambda>0$. When the couple $(X, Y)$ verifies additional properties (see [11] for details), the relative closure of $(X, Y)$ is defined as $(X, Y)_{0}=\left\{\mathbf{x} \in \mathbb{R}^{n} \mid(\mathbf{x}, 0) \in \bar{X} \backslash \bar{Y}\right\}$, where $\bar{X}$ is the topological closure of $X$. In the special case where $Y=\varnothing$, we denote the relative closure by $X_{0}$. When $Y$ is empty, the restrictions put on couples imply that the fibers $X_{\lambda}$ are compact, and $X_{0}$ is then simply the Hausdorff limit of $X_{\lambda}$ as $\lambda$ goes to zero.

Theorem 1 is applicable in this special case, and effective estimates can be derived as in the algebraic case, since, as in the case of Corollary 2, the set $D_{a}^{p}(\delta)$ is given by a quantifier-free formula. We obtain

Corollary 3. Let $X \subseteq \mathbb{R}^{n} \times \mathbb{R}_{+}$be a bounded semi-Pfaffian set such that the fiber $X_{\lambda}$ is compact for all $\lambda>0$, and let $X_{0}$ be the relative closure of $X$. If for $\lambda$ small 
enough, the format of $X_{\lambda}$ is bounded componentwise by $(n, \ell, \alpha, \beta, s)$, we have for any integer $k$,

$$
b_{k}\left(X_{0}\right) \leq 2^{\ell^{2}(k+1)^{2} / 2} s^{2 n(k+1)} O(k n(\alpha+\beta))^{(k+1)(n+\ell)} .
$$

Remark 4. Theorem 1 can also be used to derive estimates on the Betti numbers of a general relative closure $(X, Y)_{0}$, where $Y$ is not empty (and thus, $(X, Y)_{0}$ is not necessarily compact). This fact is proved in [29], and good upper-bounds for that case is the subject of a separate paper.

\section{Organization of the Paper}

The rest of the paper is organized as follows: in Section 1 we reduce the problem of the Hausdorff limit of a sequence in a definable family $A \subseteq \mathbb{R}^{n+r}$ to the case of the Hausdorff limit $X_{0}$ of a one-parameter family $X \subseteq \mathbb{R}^{n} \times \mathbb{R}_{+}$when the parameter $\lambda$ goes to zero. We then describe the ingredients of the proof of Theorem 1 for that case: we need to construct a family of continuous surjections $f^{\lambda}: X_{\lambda} \rightarrow X_{0}$. Using the spectral sequence associated to such a surjection, we can estimate the Betti numbers of $X_{0}$ in terms of the Betti numbers of the fibered products of $X_{\lambda}$. Such fibered products then need to be approximated to obtain an estimate in terms of the Betti numbers of expanded diagonals $D_{\lambda}^{p}(\delta)$

In Section 2 the family $f^{\lambda}$ is constructed using definable triangulations of functions. We prove two important properties of this family: $f^{\lambda}$ is close to identity when $\lambda$ goes to zero and, for any $\lambda^{\prime} \neq \lambda$, we can obtain $f^{\lambda}$ by composing $f^{\lambda^{\prime}}$ with a homeomorphism $h: X_{\lambda} \rightarrow X_{\lambda^{\prime}}$ (see Proposition 8).

Section 3 is devoted to the topological approximations that lead to Theorem 1. In Section 4 the algebraic and Pfaffian complexity estimates (Corollaries 2 and 3 ) are proved. The section also contains all the relevant background material on Pfaffian functions and on Betti numbers of quantifier-free formulas.

\section{Reduction to One Parameter and Strategy}

In this section we show how, using the results of Lion and Speissegger on the definability of Hausdorff limits [21], we can reduce the general case of a Hausdorff limit that occurs in a family with $r$ parameters to the case where $r=1$.

Fix an o-minimal expansion $\mathcal{S}$ of the real field (see [7] or [8]). Let $A \subseteq \mathbb{R}^{n+r}$ be a bounded definable set with compact fibers, and let $L$ be the Hausdorff limit of a sequence of fibers of $A$. We assume of course that $L$ is not already a fiber of $A$, since Theorem 1 is trivial in this case. Since sequences of parameters $\left(a_{i}\right)$ in $A^{\prime}$ are not definable in $\mathcal{S}$, it is difficult to handle Hausdorff limits directly. To avoid this problem, Lion and Speissegger constructed in [21] a new family $B$ to model the Hausdorff limits of fibers of $A$. The main result they prove is the following.

Theorem 5 [21]. If $A \subseteq \mathbb{R}^{n+r}$ is a bounded definable set with compact fibers, there 
exists $R \geq r$ and a definable compact $B \subseteq \mathbb{R}^{n+R}$ such that, if $B^{\prime}$ is the projection of $B$ on $\mathbb{R}^{R}$, the following properties hold:

(H1) For every $a \in A^{\prime}$, there is $a b \in B^{\prime}$ such that $A_{a}=B_{b}$;

(H2) for every sequence $\left(b_{i}\right)$ in $B^{\prime}$ such that $\lim b_{i}=b^{*}$, the Hausdorff limit of $B_{b_{i}}$ exists and equals $B_{b^{*}}$

(H3) $\operatorname{dim} B^{\prime}=\operatorname{dim} A^{\prime}$ and $\operatorname{dim}\left\{b \in B^{\prime} \mid \forall a \in A^{\prime}, B_{b} \neq A_{a}\right\}<\operatorname{dim} A^{\prime}$.

The proof of this result is quite technical, and involves representing the fibers of $A$ and their possible Hausdorff limits in terms of integral manifolds of some distributions that depend only on $A$.

Using Theorem 5, we can obtain $L$ as a limit of a one-parameter family by the following proposition.

Proposition 6. Let $A \subseteq \mathbb{R}^{n+r}$ be a bounded definable set with compact fibers, and let $L$ be the Hausdorff limit of a sequence $A_{a_{i}}$. Then there exists a definable family $X \subseteq \mathbb{R}^{n} \times(0,1)$ such that the following hold:

(X1) For every $\lambda \in(0,1)$, there exists $a(\lambda) \in A^{\prime}$ such that $X_{\lambda}=A_{a(\lambda)}$.

(X2) $L$ is the Hausdorff limit $X_{0}$ of $X_{\lambda}$ when $\lambda$ goes to zero.

Proof. Let $B$ be the set described in Theorem 5. By property (H1), the set of parameters $B^{\prime}$ contains a sequence $b_{i}$ such that $A_{a_{i}}=B_{b_{i}}$ for all $i$. Since $B^{\prime}$ is compact, we can assume by taking a subsequence that $b_{i}$ converges to some $b^{*} \in B^{\prime}$. By property (H2), we must have $L=B_{b^{*}}$, since the Hausdorff limit is unique. Since $A_{a_{i}}=B_{b_{i}}$ for all $i$, the point $b^{*}$ is in the closure of the definable set

$$
C=\left\{b \in B^{\prime} \mid \exists a \in A^{\prime}, A_{a}=B_{b}\right\} .
$$

By the curve selection lemma [8, Chapter 6, Corollary 1.5], there exists a definable curve $\gamma:(0,1) \rightarrow C$ such that $\lim _{\lambda \rightarrow 0} \gamma(\lambda)=b^{*}$. Consider the definable family $X$ given by

$$
X=\left\{(\mathbf{x}, \lambda) \in \mathbb{R}^{n} \times(0,1) \mid \mathbf{x} \in B_{\gamma(\lambda)}\right\} .
$$

Since $\gamma(\lambda) \in C$ for all $\lambda \in(0,1)$, there exists for each $\lambda$ a point $a(\lambda) \in A^{\prime}$ such that $B_{\gamma(\lambda)}=A_{a(\lambda)}$, so property (X1) holds. Moreover, property (H2) in Theorem 5 guarantees that the Hausdorff limit of $B_{\gamma(\lambda)}$ when $\lambda$ goes to zero is $B_{b^{*}}$, and since $B_{b^{*}}=L$ by construction, (X2) holds too.

Throughout the rest of this paper, we assume we are given $X$ as in Proposition 6, with Hausdorff limit $X_{0}$ when $\lambda$ goes to zero. The strategy will be the following. Using the fact that $\bar{X}$ is definable and compact, and thus that the projection $\pi$ of $\bar{X}$ on the $\lambda$-axis can be triangulated, we construct in Section 2 a family of continuous surjections $f^{\lambda}: X_{\lambda} \rightarrow X_{0}$ defined for small values of $\lambda$. Since $X_{\lambda}$ and $X_{0}$ are both compact, the surjection $f^{\lambda}$ is closed, and we can apply the following theorem [15, Theorem 1]. 
Theorem 7. Let $f: X \rightarrow X_{0}$ be a closed continuous surjective map definable in an $o$-minimal structure. For all integers $k$, the following inequality holds:

$$
b_{k}\left(X_{0}\right) \leq \sum_{p+q=k} b_{q}\left(W^{p}\right),
$$

where $W^{p}$ is the $(p+1)$-fold fibered product of $X$;

$$
W^{p}=\left\{\left(\mathbf{x}_{0}, \ldots, \mathbf{x}_{p}\right) \in X^{p+1} \mid f\left(\mathbf{x}_{0}\right)=\cdots=f\left(\mathbf{x}_{p}\right)\right\} .
$$

Thus, the existence of $f^{\lambda}$ for a fixed $\lambda>0$ gives estimates on the Betti numbers of $X_{0}$ in terms of the Betti numbers of the definable sets $W_{\lambda}^{p}$ (obtained by taking $f$ to be $f^{\lambda}$ in Theorem 7). However, there is no explicit description of these fibered products in the general case, so this fact alone is not sufficient to establish effective upper-bounds in the algebraic and Pfaffian case.

Section 3 is devoted to refining the estimate given by the spectral sequence to finally obtain Theorem 1, which gives an estimate for the Betti numbers of $X_{0}$ in terms of definable sets that are described in a completely explicit way: the expanded diagonals $D_{\lambda}^{p}(\delta)$. The result is achieved by showing that for suitable values of $\delta$ and $\lambda$, the fibered product $W_{\lambda}^{p}$ is included in the expanded diagonal $D_{\lambda}^{p}(\delta)$, and that this inclusion induces an isomorphism between the corresponding homology groups (Proposition 22).

\section{Construction of a Family of Surjections}

The setting for this section is the following: we consider a definable family $X \subseteq \mathbb{R}^{n} \times$ $(0,1)$ such that the fiber $X_{\lambda}$ is compact for all $\lambda \in(0,1)$ and such that this family has a Hausdorff limit $X_{0}$ when $\lambda$ goes to zero. As announced in the previous section, we will construct for small values of $\lambda$ a family of continuous surjections $f^{\lambda}: X_{\lambda} \rightarrow X_{0}$ that are close to identity. More precisely, we will prove the following result.

Proposition 8. Let $X$ be a definable family as above. There exists $\lambda_{0}>0$ and a family of definable continuous surjections $f^{\lambda}: X_{\lambda} \rightarrow X_{0}$, defined for all $\lambda \in\left(0, \lambda_{0}\right)$ such that

$$
\lim _{\lambda \rightarrow 0} \max _{\mathbf{x} \in X_{\lambda}}\left|\mathbf{x}-f^{\lambda}(\mathbf{x})\right|=0 .
$$

Moreover, this family $f^{\lambda}$ verifies the following property: for all $0<\lambda^{\prime}<\lambda<\lambda_{0}$, there exists a (uniformly) definable homeomorphism $h: X_{\lambda} \rightarrow X_{\lambda^{\prime}}$ such that for all $\mathbf{x} \in X_{\lambda}$, we have $f^{\lambda}(\mathbf{x})=f^{\lambda^{\prime}}(h(\mathbf{x}))$.

\subsection{Triangulation of the Projection on $\lambda$}

Since the terminology concerning simplexes is somewhat variable, we now state the precise definitions we use. These definitions follow [7] rather than [8]. 
Definition 9. If $\boldsymbol{\alpha}_{0}, \ldots, \boldsymbol{\alpha}_{d}$ are affine-independent points in $\mathbb{R}^{n}$, the closed simplex $\bar{\sigma}=\left[\boldsymbol{\alpha}_{0}, \ldots, \boldsymbol{\alpha}_{d}\right]$ is the subset of $\mathbb{R}^{n}$ defined by

$$
\bar{\sigma}=\left\{\sum_{i=0}^{d} w_{i} \boldsymbol{\alpha}_{i} \mid \sum_{i=0}^{d} w_{i}=1, w_{1} \geq 0, \ldots, w_{d} \geq 0\right\} .
$$

A function $g: \bar{\sigma} \rightarrow \mathbb{R}$ is affine if it satisfies the equality

$$
g\left(\sum_{i=0}^{d} w_{i} \boldsymbol{\alpha}_{i}\right)=\sum_{i=0}^{d} w_{i} g\left(\boldsymbol{\alpha}_{i}\right)
$$

for any $w_{0}, \ldots, w_{d}$ as in (8).

A face of $\bar{\sigma}$ is any closed simplex obtained from a non-empty subset of $\boldsymbol{\alpha}_{0}, \ldots, \boldsymbol{\alpha}_{d}$. The open simplex $\sigma=\left(\boldsymbol{\alpha}_{0}, \ldots, \boldsymbol{\alpha}_{d}\right)$ is the subset of points $\sum_{i=0}^{d} w_{i} \boldsymbol{\alpha}_{i}$ in $\bar{\sigma}$ for which $w_{i}>0$ for all $0 \leq i \leq d$.

Definition 10. A (finite) simplicial complex $K$ of $\mathbb{R}^{n}$ is a finite collection $\left\{\bar{\sigma}_{1}, \ldots, \bar{\sigma}_{k}\right\}$ of closed simplexes that is closed under taking faces, and such that $\bar{\sigma}_{i} \cap \bar{\sigma}_{j}$ is a common face of $\bar{\sigma}_{i}$ and $\bar{\sigma}_{j}$ for any $1 \leq i, j \leq k$. The geometric realization of $K$ is the subset of $\mathbb{R}^{n}$ defined by $|K|=\bar{\sigma}_{1} \cup \cdots \cup \bar{\sigma}_{k}$.

Throughout Section 2 , we denote by $\pi: \bar{X} \rightarrow \mathbb{R}$ the projection on the $\lambda$-coordinate. Note that $\bar{X}$ is definable (since it is the closure of a definable set, see for instance Proposition 1.12 of [7]), so $\pi$ is definable too (i.e. the graph of $\pi$ is a definable set). Since $\bar{X}$ is also compact, the triangulation theorem for definable functions (see [6] or [7]) allows us to assume without loss of generality that $\bar{X}$ is the geometric realization of a simplicial complex $K$ and that the map $\pi$ is affine on each simplex $\bar{\sigma}$ of $K$. (Note that, in general, this requires a linear change of coordinates in the fibers, but this does not affect our results.) Moreover, we identify $X_{0}$ with $X_{0} \times\{0\} \subseteq X$ and we assume that the triangulation has been refined so that it is compatible with $X_{0}$, i.e. $X_{0}$ is the union of open simplices of the triangulation.

In the present section we need to consider points in both the total space $\bar{X} \subseteq \mathbb{R}^{n} \times[0,1]$ and points in fibers $X_{\lambda}$, which are by definition subspaces of $\mathbb{R}^{n}$. To avoid ambiguities, we use the following convention: bold Greek letters such as $\boldsymbol{\xi}$ denote points in the total space, whereas bold Roman letters are used to denote the points in fibers $X_{\lambda}$. Thus, if $\pi(\boldsymbol{\xi})=\lambda>0$, we have $\boldsymbol{\xi}=(\mathbf{x}, \lambda)$, where $\mathbf{x} \in X_{\lambda}$.

Definition 11. The star $S$ of $X_{0}$ in $X$ is the union of $X_{0}$ with all the open simplices $\left(\boldsymbol{\alpha}_{0}, \ldots, \boldsymbol{\alpha}_{d}\right)$ that have at least one vertex $\boldsymbol{\alpha}_{i}$ in $X_{0}$.

\subsection{Constructing a Retraction}

Let us define the retraction $F: S \rightarrow X_{0}$ as follows. If $\boldsymbol{\xi} \in X_{0}$, we let $F(\boldsymbol{\xi})=\boldsymbol{\xi}$. If $\boldsymbol{\xi}$ belongs to some open simplex $\sigma=\left(\boldsymbol{\alpha}_{0}, \ldots, \boldsymbol{\alpha}_{d}\right)$, where $\boldsymbol{\alpha}_{0}, \ldots, \boldsymbol{\alpha}_{d}$ are vertices such 
that $\boldsymbol{\alpha}_{0}, \ldots, \boldsymbol{\alpha}_{k}$ are in $X_{0}$ and $\boldsymbol{\alpha}_{k+1}, \ldots, \boldsymbol{\alpha}_{d}$ are not in $X_{0}$, for some $k$ with $0 \leq k<d$, define $F$ on $\sigma$ by

$$
F\left(\sum_{i=0}^{d} w_{i} \boldsymbol{\alpha}_{i}\right)=\frac{1}{\sum_{i=0}^{k} w_{i}} \sum_{i=0}^{k} w_{i} \boldsymbol{\alpha}_{i} .
$$

If $\boldsymbol{\xi}=(\mathbf{x}, \lambda) \in S$ with $\lambda>0$, we denote by $\Delta(\boldsymbol{\xi})$ the intersection between the line through $\boldsymbol{\xi}$ and $F(\boldsymbol{\xi})$ and the unique open simplex $\sigma$ containing $\boldsymbol{\xi}$.

Proposition 12. The above definition gives a continuous retraction $F: S \rightarrow X_{0}$ that verifies: for all $\boldsymbol{\xi}=(\mathbf{x}, \lambda) \in S$ with $\lambda>0$ and all $\boldsymbol{\zeta} \in \Delta(\boldsymbol{\xi})$, we have $F(\boldsymbol{\zeta})=F(\boldsymbol{\xi})$.

Proof. Let $\sigma=\left(\boldsymbol{\alpha}_{0}, \ldots, \boldsymbol{\alpha}_{d}\right)$ be an open simplex, with $\boldsymbol{\alpha}_{0}, \ldots, \boldsymbol{\alpha}_{k}$ in $X_{0}$ and $\boldsymbol{\alpha}_{k+1}, \ldots$, $\boldsymbol{\alpha}_{d}$ not in $X_{0}$, for some $k$ with $0 \leq k<d$, so that $\sigma \subseteq S$. Fix $\boldsymbol{\xi}=\sum_{i=0}^{d} w_{i} \boldsymbol{\alpha}_{i}$ in $\sigma$, and let $s=\sum_{i=0}^{k} w_{i}$. Since all the weights $w_{i}$ are positive, the inequality $0 \leq k<d$ implies that $0<s<1$. Thus, formula (10) clearly defines a continuous function from $\sigma$ to $X_{0}$. Moreover, if $\sigma^{\prime}=\left(\boldsymbol{\alpha}_{i_{0}}, \ldots, \boldsymbol{\alpha}_{i_{e}}\right)$ is a face of $\sigma$ with at least one $0 \leq j \leq e$ such that $i_{j} \leq k$ (so that $\sigma^{\prime} \subseteq S$ ), it is clear that expression (10) extends $F$ continuously to $\sigma^{\prime}$.

If $\sigma$ and $\boldsymbol{\xi}$ are as above and $\boldsymbol{\zeta}=t \boldsymbol{\xi}+(1-t) F(\boldsymbol{\xi})$ is a point on $\Delta(\boldsymbol{\xi})$, we now show that $F(\boldsymbol{\xi})=F(\boldsymbol{\zeta})$. We have $\boldsymbol{\zeta}=\sum_{i=0}^{k} w_{i}^{\prime} \boldsymbol{\alpha}_{i}$, where

$$
w_{i}^{\prime}= \begin{cases}t w_{i}+(1-t) \frac{w_{i}}{s} & \text { if } \quad 0 \leq i \leq k \\ t w_{i} & \text { if } \quad k+1 \leq i \leq d\end{cases}
$$

To prove that $F(\boldsymbol{\xi})=F(\boldsymbol{\zeta})$, we must prove that for all $0 \leq i \leq k$,

$$
\frac{w_{i}}{\sum_{j=0}^{k} w_{j}}=\frac{w_{i}^{\prime}}{\sum_{j=0}^{k} w_{j}^{\prime}}
$$

Cross-multiplying, we get the following quantities:

$$
w_{i} \sum_{j=0}^{k} w_{j}^{\prime}=w_{i} \sum_{j=0}^{k}\left(t w_{j}+(1-t) \frac{w_{j}}{s}\right)=w_{i}(1-t+t s)
$$

and

$$
w_{i}^{\prime} \sum_{j=0}^{k} w_{j}=\left(t w_{i}+(1-t) \frac{w_{i}}{s}\right) s=(t s+(1-t)) w_{i} .
$$

The two final expressions in (11) and (12) are clearly equal, so $F(\boldsymbol{\zeta})=F(\boldsymbol{\xi})$ for any $\zeta \in \Delta(\xi)$.

Definition 13. Let $\lambda_{0}=\min \left\{\pi(\boldsymbol{\alpha}) \mid \boldsymbol{\alpha}\right.$ is a vertex of $\left.X, \boldsymbol{\alpha} \notin X_{0}\right\}$. For any $\lambda \in$ $\left(0, \lambda_{0}\right)$, we define $f^{\lambda}: X_{\lambda} \rightarrow X_{0}$ by $f^{\lambda}(\mathbf{x})=F(\mathbf{x}, \lambda)$.

Since $\pi(\boldsymbol{\alpha})=0$ can only happen if $\boldsymbol{\alpha} \in X_{0}$, it follows that $\lambda_{0}>0$. Note also that if $\boldsymbol{\xi}=(\mathbf{x}, \lambda)$ is not in $S$, then we must have $\lambda \geq \lambda_{0}$. Indeed, if $\boldsymbol{\xi} \notin S$, it belongs to an 
open simplex $\sigma$ of the form $\left(\boldsymbol{\alpha}_{0}, \ldots, \boldsymbol{\alpha}_{d}\right)$ such that none of the vertices is in $X_{0}$. Thus, $\pi\left(\boldsymbol{\alpha}_{i}\right) \geq \lambda_{0}$ for all $i$, and since $\pi$ is affine on $\sigma$, we must have $\lambda=\pi(\boldsymbol{\xi}) \geq \lambda_{0}$ too. Thus, for any fixed $\lambda \in\left(0, \lambda_{0}\right)$, we have $\left\{(\mathbf{x}, \lambda) \mid \mathbf{x} \in X_{\lambda}\right\} \subseteq S$, so $f^{\lambda}$ is well-defined for $\lambda \in\left(0, \lambda_{0}\right)$. Since $F$ is continuous, $f^{\lambda}$ is continuous too.

\subsection{Properties of the Maps $f^{\lambda}$}

We must still show that the family of mappings $f^{\lambda}$ has all the properties described in Proposition 8: $f^{\lambda}$ is surjective (Lemma 14), close to identity (Proposition 15), and $f^{\lambda}$ can be obtained from $f^{\lambda^{\prime}}$ by composing on the right by a homeomorphism $h: X_{\lambda} \rightarrow X_{\lambda^{\prime}}$ (Proposition 16).

Lemma 14. For all $\lambda \in\left(0, \lambda_{0}\right)$, the map $f^{\lambda}$ is surjective.

Proof. Let $\boldsymbol{\zeta} \in X_{0}$. Then there exists a unique set of vertices $\left\{\boldsymbol{\alpha}_{0}, \ldots, \boldsymbol{\alpha}_{k}\right\}$ such that $\boldsymbol{\zeta}$ belongs to the open simplex $\left(\boldsymbol{\alpha}_{0}, \ldots, \boldsymbol{\alpha}_{k}\right)$; let $v_{0}, \ldots, v_{k}$ be the corresponding weights, so that $\boldsymbol{\zeta}=\sum_{i=0}^{k} v_{i} \boldsymbol{\alpha}_{i}$. There must be vertices $\boldsymbol{\alpha}_{k+1}, \ldots, \boldsymbol{\alpha}_{d}$ such that the open simplex $\sigma=\left(\boldsymbol{\alpha}_{0}, \ldots, \boldsymbol{\alpha}_{d}\right)$ is in $X$, otherwise $\boldsymbol{\zeta}$ could not be approximated by points of $X_{\lambda}$ for $\lambda>0$.

Let $\boldsymbol{\xi}=\sum_{i=0}^{d} w_{i} \boldsymbol{\alpha}_{i}$ where $w_{i}=v_{i} / 2$ for $0 \leq i \leq k$ and $w_{k+1}, \ldots, w_{d}$ are arbitrarily chosen positive numbers so that $\sum_{i=0}^{d} w_{i}=1$. By choice of $w_{0}, \ldots, w_{k}$, we have $\sum_{i=0}^{k} w_{i}=\frac{1}{2}$, and thus $F(\boldsymbol{\xi})=\boldsymbol{\zeta}$. Moreover, if $\Delta(\boldsymbol{\xi})$ is as defined in Proposition 12, there must be a point $\tau \in \Delta(\xi)$ such that $\pi(\tau)=\lambda$. Indeed, if we parameterize the line between $\boldsymbol{\xi}$ and $\zeta$ by $\{(1-t) \boldsymbol{\zeta}+t \boldsymbol{\xi} \mid t \in \mathbb{R}\}$, the endpoints of $\Delta(\boldsymbol{\xi})$ are obtained for $t=0$ and $t=2$, which give respectively the points $\zeta$ and $\zeta^{\prime}=2 \sum_{i=k+1}^{d} w_{i} \boldsymbol{\alpha}_{i}$. We have $\pi(\zeta)=0$, and since $\zeta^{\prime}$ is not in $S$, we have $\pi\left(\zeta^{\prime}\right) \geq \lambda_{0}$. Since by restriction $\pi$ is affine on $\Delta(\xi), \pi(\tau)$ takes all the values in the interval $\left(0, \pi\left(\boldsymbol{\zeta}^{\prime}\right)\right)$ when $\tau$ runs through $\Delta(\boldsymbol{\xi})$. In particular, if $\lambda<\lambda_{0}$ there exists $\tau \in \Delta(\boldsymbol{\xi})$ with $\pi(\tau)=\lambda$. By Proposition 12, we must have $F(\tau)=F(\xi)$ and since $F(\boldsymbol{\xi})=\boldsymbol{\zeta}$, this proves that $f^{\lambda}$ is surjective.

Proposition 15. For $f^{\lambda}$ as in Definition 13, we have

$$
\lim _{\lambda \rightarrow 0} \max _{\mathbf{x} \in X_{\lambda}}\left|\mathbf{x}-f^{\lambda}(\mathbf{x})\right|=0 .
$$

Proof. Let $\sigma=\left(\boldsymbol{\alpha}_{0}, \ldots, \boldsymbol{\alpha}_{d}\right)$ be an open simplex where $\boldsymbol{\alpha}_{0}, \ldots, \boldsymbol{\alpha}_{k}$ are in $X_{0}$ and $\boldsymbol{\alpha}_{k+1}, \ldots, \boldsymbol{\alpha}_{d}$ are not in $X_{0}$, where $0 \leq k<d$. Fix $\boldsymbol{\xi}=\sum_{i=0}^{d} w_{i} \boldsymbol{\alpha}_{i}$ in $\sigma$, and let $s=\sum_{i=0}^{k} w_{i}$. We have

$$
\sum_{i=k+1}^{d} w_{i}=\sum_{i=0}^{d} w_{i}-\sum_{i=0}^{k} w_{i}=1-s
$$

and

$$
\boldsymbol{\xi}-F(\boldsymbol{\xi})=\sum_{i=0}^{d} w_{i} \boldsymbol{\alpha}_{i}-\frac{1}{s} \sum_{i=0}^{k} w_{i} \boldsymbol{\alpha}_{i}=\left(1-\frac{1}{s}\right)\left(\sum_{i=0}^{k} w_{i} \boldsymbol{\alpha}_{i}\right)+\sum_{i=k+1}^{d} w_{i} \boldsymbol{\alpha}_{i} .
$$


By the triangle inequality, we obtain

$$
|\boldsymbol{\xi}-F(\boldsymbol{\xi})| \leq \max _{o \leq i \leq d}\left|\boldsymbol{\alpha}_{i}\right|\left(\left|1-\frac{1}{s}\right|\left(\sum_{i=0}^{k} w_{i}\right)+\sum_{i=k+1}^{d} w_{i}\right)=2(1-s) \max _{0 \leq i \leq d}\left|\boldsymbol{\alpha}_{i}\right| .
$$

Let $\lambda=\pi(\boldsymbol{\xi})=\sum_{i=k+1}^{d} w_{i} \pi\left(\boldsymbol{\alpha}_{i}\right)$. Since $\pi\left(\boldsymbol{\alpha}_{i}\right) \geq \lambda_{0}$ for all $i \geq k+1$, it follows that

$$
\lambda=\sum_{i=k+1}^{d} w_{i} \pi\left(\boldsymbol{\alpha}_{i}\right) \geq \lambda_{0}\left(\sum_{i=k+1}^{d} w_{i}\right)=\lambda_{0}(1-s) .
$$

It follows that $1-s \leq \lambda / \lambda_{0}$. Combining this with (14), we obtain

$$
|\boldsymbol{\xi}-F(\boldsymbol{\xi})| \leq 2 \frac{\lambda}{\lambda_{0}} \max _{0 \leq i \leq d}\left|\boldsymbol{\alpha}_{i}\right| \leq 2 \frac{\lambda}{\lambda_{0}} \max \{|\boldsymbol{\alpha}|, \boldsymbol{\alpha} \text { vertex of } K\} .
$$

Thus, $|\boldsymbol{\xi}-F(\boldsymbol{\xi})|$ is bounded by a quantity independent of $\boldsymbol{\xi}$ that goes to zero when $\lambda$ goes to zero, and since $\left|\mathbf{x}-f^{\lambda}(\mathbf{x})\right| \leq|\boldsymbol{\xi}-F(\boldsymbol{\xi})|$, the result follows.

Proposition 16. For all $0<\lambda^{\prime}<\lambda<\lambda_{0}$, there exists a homeomorphism $h$ : $X_{\lambda} \rightarrow X_{\lambda^{\prime}}$ such that $f^{\lambda}=f^{\lambda^{\prime}} \circ h$.

Proof. Let $\boldsymbol{\xi} \in X_{\lambda}$, and let $\Delta(\xi)$ be as in Proposition 12. Since $\pi$ is affine on $\Delta(\xi)$, if $\boldsymbol{\tau}=t \boldsymbol{\xi}+(1-t) F(\boldsymbol{\xi})$ is a point on $\Delta(\boldsymbol{\xi})$, we have

$$
\pi(\boldsymbol{\tau})=t \pi(\boldsymbol{\xi})+(1-t) \pi(F(\boldsymbol{\xi}))=t \lambda .
$$

Thus, $\tau \in X_{\lambda^{\prime}}$ if and only if $t=\lambda^{\prime} / \lambda$, and so the map $h$ defined by

$$
h(\boldsymbol{\xi})=\frac{\lambda^{\prime}}{\lambda} \boldsymbol{\xi}+\left(1-\frac{\lambda^{\prime}}{\lambda}\right) F(\boldsymbol{\xi})
$$

maps $X_{\lambda}$ to $X_{\lambda^{\prime}}$.

Suppose that there exists $\boldsymbol{\xi}$ and $\boldsymbol{\xi}^{\prime}$ in $X_{\lambda}$ such that $h(\xi)=h\left(\boldsymbol{\xi}^{\prime}\right)=\boldsymbol{\tau}$. Then $\boldsymbol{\tau} \in \Delta(\boldsymbol{\xi})$ and $\boldsymbol{\tau} \in \Delta\left(\boldsymbol{\xi}^{\prime}\right)$, and by Proposition 12 this means that $F(\boldsymbol{\xi})=F(\boldsymbol{\tau})=F\left(\boldsymbol{\xi}^{\prime}\right)$. Then (16) implies that $\boldsymbol{\xi}=\boldsymbol{\xi}^{\prime}$, so $h$ is injective. The map $h$ is also surjective, since for $\boldsymbol{\tau} \in X_{\lambda^{\prime}}$, it is easy to verify that the point $\boldsymbol{\xi}$ defined by

$$
\xi=\frac{\lambda}{\lambda^{\prime}} \boldsymbol{\tau}-\left(\frac{\lambda}{\lambda^{\prime}}-1\right) F(\tau)
$$

is a point in $X_{\lambda}$ such that $h(\xi)=\tau$.

The continuity of $h$ follows from the continuity of $F$. Since $h(\xi) \in \Delta(\xi)$ by construction, Proposition 12 implies that $F(h(\xi))=F(\xi)$, so $f^{\lambda}=f^{\lambda^{\prime}} \circ h$.

\section{Approximation of the Fibered Products}

We now turn our attention to the fibered products associated to the surjections $f^{\lambda}: X_{\lambda} \rightarrow$ $X_{0}$ that were constructed in the previous section. We prove in Proposition 22 the approximation result for those sets that yields the result of the main theorem. 
Define for $p \in \mathbb{N}$ and $\lambda \in\left(0, \lambda_{0}\right)$,

$$
W_{\lambda}^{p}=\left\{\left(\mathbf{x}_{0}, \ldots, \mathbf{x}_{p}\right) \in\left(X_{\lambda}\right)^{p+1} \mid f^{\lambda}\left(\mathbf{x}_{0}\right)=\cdots=f^{\lambda}\left(\mathbf{x}_{p}\right)\right\} .
$$

From Theorem 7 we have for any $\lambda \in\left(0, \lambda_{0}\right)$,

$$
b_{k}\left(X_{0}\right) \leq \sum_{p+q=k} b_{q}\left(W_{\lambda}^{p}\right) .
$$

Thus, bounding the Betti numbers of $X_{0}$ can be reduced to estimating the Betti numbers of the sets $W_{\lambda}^{p}$ for some $\lambda \in\left(0, \lambda_{0}\right)$. The first step in that direction is the following.

Proposition 17. For all $0<\lambda^{\prime}<\lambda<\lambda_{0}$, the sets $W_{\lambda}^{p}$ and $W_{\lambda^{\prime}}^{p}$ are homeomorphic.

Proof. Fix $0<\lambda^{\prime}<\lambda<\lambda_{0}$, and let $h$ be the homeomorphism between $X_{\lambda}$ and $X_{\lambda^{\prime}}$ described in Proposition 16. Since $f^{\lambda^{\prime}} \circ h=f^{\lambda}$, the map $h^{p}:\left(X_{\lambda}\right)^{p+1} \rightarrow\left(X_{\lambda^{\prime}}\right)^{p+1}$ defined by

$$
h^{p}\left(\mathbf{x}_{0}, \ldots, \mathbf{x}_{p}\right)=\left(h\left(\mathbf{x}_{0}\right), \ldots, h\left(\mathbf{x}_{p}\right)\right)
$$

maps $W_{\lambda}^{p}$ homeomorphically onto $W_{\lambda^{\prime}}^{p}$.

Recall that for $p \in \mathbb{N}$ and $\mathbf{x}_{0}, \ldots, \mathbf{x}_{p} \in \mathbb{R}^{n}, \rho_{p}$ is the polynomial

$$
\rho_{p}\left(\mathbf{x}_{0}, \ldots, \mathbf{x}_{p}\right)=\sum_{0 \leq i<j \leq p}\left|\mathbf{x}_{i}-\mathbf{x}_{j}\right|^{2} .
$$

For $\lambda \in\left(0, \lambda_{0}\right), \varepsilon>0$ and $\delta>0$, we define the following sets:

$$
\begin{aligned}
W_{\lambda}^{p}(\varepsilon) & =\left\{\left(\mathbf{x}_{0}, \ldots, \mathbf{x}_{p}\right) \in\left(X_{\lambda}\right)^{p+1} \mid \rho_{p}\left(f^{\lambda}\left(\mathbf{x}_{0}\right), \ldots, f^{\lambda}\left(\mathbf{x}_{p}\right)\right) \leq \varepsilon\right\}, \\
D_{\lambda}^{p}(\delta) & =\left\{\left(\mathbf{x}_{0}, \ldots, \mathbf{x}_{p}\right) \in\left(X_{\lambda}\right)^{p+1} \mid \rho_{p}\left(\mathbf{x}_{0}, \ldots, \mathbf{x}_{p}\right) \leq \delta\right\} .
\end{aligned}
$$

Proposition 18. Let $p \in \mathbb{N}$ be fixed. There exists $\varepsilon_{0}>0$, such that for all $\lambda \in\left(0, \lambda_{0}\right)$ and all $0<\varepsilon^{\prime}<\varepsilon<\varepsilon_{0}$, the inclusion $W_{\lambda}^{p}\left(\varepsilon^{\prime}\right) \hookrightarrow W_{\lambda}^{p}(\varepsilon)$ is a homotopy equivalence. In particular, this implies that

$$
b_{q}\left(W_{\lambda}^{p}(\varepsilon)\right)=b_{q}\left(W_{\lambda}^{p}\right)
$$

for all integer $q$, all $\lambda \in\left(0, \lambda_{0}\right)$ and all $\varepsilon \in\left(0, \varepsilon_{0}\right)$.

Proof. First, notice that it is enough to prove the result for a fixed $\lambda \in\left(0, \lambda_{0}\right)$, since if $0<\lambda^{\prime}<\lambda<\lambda_{0}$ are fixed, the map $h^{p}$ introduced in (19) induces a homeomorphism between $W_{\lambda}^{p}(\varepsilon)$ and $W_{\lambda^{\prime}}^{p}(\varepsilon)$ for any $\varepsilon>0$.

Fix $\lambda \in\left(0, \lambda_{0}\right)$. By the generic triviality theorem (see Chapter 9, Theorem 1.2, of [8] or Theorem 5.22 of [7]), there exists $\varepsilon_{0}>0$ such that the projection

$$
\left\{\left(\mathbf{x}_{0}, \ldots, \mathbf{x}_{p}, \varepsilon\right) \mid \varepsilon \in\left(0, \varepsilon_{0}\right) \text { and }\left(\mathbf{x}_{0}, \ldots, \mathbf{x}_{p}\right) \in W_{\lambda}(\varepsilon)\right\} \mapsto \varepsilon
$$

is a trivial fibration. It follows that for all $0<\varepsilon^{\prime}<\varepsilon<\varepsilon_{0}$, the inclusion $W_{\lambda}^{p}\left(\varepsilon^{\prime}\right) \hookrightarrow$ $W_{\lambda}^{p}(\varepsilon)$ is a homotopy equivalence, which proves the first part of the proposition. 
The inclusions $W_{\lambda}^{p}\left(\varepsilon^{\prime}\right) \hookrightarrow W_{\lambda}^{p}(\varepsilon)$ for $\varepsilon^{\prime}<\varepsilon$ make the family $W_{\lambda}^{p}(\varepsilon), \varepsilon>0$, into a directed system, and we have

$$
\lim _{\longleftarrow} W_{\lambda}^{p}(\varepsilon) \cong \bigcap_{\varepsilon>0} W_{\lambda}^{p}(\varepsilon)=W_{\lambda}^{p} .
$$

The induced maps in Čech homology make the family $\check{H}_{*}\left(W_{\lambda}^{p}(\varepsilon)\right), \varepsilon>0$, into a directed system too, and by the continuity property of the Čech homology [10, Chapter 10], this implies that the Čech homology groups of $W_{\lambda}^{p}$ are isomorphic to the inverse limit of the groups $\check{H}_{*}\left(W_{\lambda}^{p}(\varepsilon)\right)$. Note however that the sets $W_{\lambda}^{p}$ and $W_{\lambda}^{p}(\varepsilon)$, being compact definable sets, are homeomorphic to finite simplicial complexes, and thus their singular and Čech homologies coincide. Hence, we have for all $q$,

$$
H_{q}\left(W_{\lambda}^{p}\right) \cong \lim _{\leftarrow} H_{q}\left(W_{\lambda}^{p}(\varepsilon)\right) .
$$

Since the inclusion $W_{\lambda}^{p}\left(\varepsilon^{\prime}\right) \hookrightarrow W_{\lambda}^{p}(\varepsilon)$ is a homotopy equivalence for all $0<\varepsilon^{\prime}<\varepsilon<\varepsilon_{0}$, it induces an isomorphism in homology for the directed system $H_{q}\left(W_{\lambda}^{p}(\varepsilon)\right)$. Thus, the ranks in that system are constant and equal to the rank of the limit, yielding (21).

Example 19. Consider the family $X \subseteq \mathbb{R}^{2} \times \mathbb{R}_{+}$such that the fiber is given for all $\lambda>0$ by

$$
X_{\lambda}=\{(x, 0) \mid 0 \leq x \leq 1\} \cup\{(0, \lambda)\} \cup\{(\lambda, \lambda)\} .
$$

If we construct $f^{\lambda}$ from a triangulation as in Definition 13, we must have $f^{\lambda}(0, \lambda)=$ $f^{\lambda}(\lambda, \lambda)=0$, independently of the choice of the triangulation. However, there are other families of continuous surjections from $X_{\lambda}$ into $X_{0}$ that are close to identity. A natural choice would be for instance to take the maps $g^{\lambda}$ defined by $g^{\lambda}(x, y)=(x, 0)$, for which we still have

$$
\lim _{\lambda \rightarrow 0} \max _{(x, y) \in X_{\lambda}}\left|g^{\lambda}(x, y)-(x, y)\right|=0 .
$$

However, it is easy to check that the topological type of the corresponding sets $W_{\lambda}^{2}(\varepsilon)$ changes exactly when $\varepsilon=\lambda$, since the two connected components of $\left(X_{\lambda}\right)^{2}$ formed by the isolated points $\{(0, \lambda, \lambda, \lambda)\}$ and $\{(\lambda, \lambda, 0, \lambda)\}$ belong to $W_{\lambda}^{2}(\varepsilon)$ exactly when $\varepsilon \geq \lambda$.

Example 19 shows that finding a family of surjections close to identity is not enough to guarantee the existence of an $\varepsilon_{0}$ independent of $\lambda$ for which Proposition 18 holds. This explains why a careful construction of $f^{\lambda}$ was necessary in Section 2 . The fact that we can find $\varepsilon_{0}$ independent of $\lambda$ plays a key part in approximating the sets $W_{\lambda}^{p}$ (see Proposition 22).

Proposition 20. Let $p \in \mathbb{N}$ be fixed. There exists $\lambda_{1}$ such that $0<\lambda_{1} \leq \lambda_{0}$ and definable functions $\delta_{0}(\lambda)$ and $\delta_{1}(\lambda)$ defined for $\lambda \in\left(0, \lambda_{1}\right)$ such that $\lim _{\lambda \rightarrow 0} \delta_{0}(\lambda)=0$, $\lim _{\lambda \rightarrow 0} \delta_{1}(\lambda) \neq 0$, and such that for all $0<\delta_{0}(\lambda)<\delta^{\prime}<\delta<\delta_{1}(\lambda)$, the inclusion $D_{\lambda}^{p}\left(\delta^{\prime}\right) \hookrightarrow D_{\lambda}^{p}(\delta)$ is a homotopy equivalence. 
Proof. Let $\lambda \in\left(0, \lambda_{0}\right)$ be fixed. As in the proof of Proposition 18, we can apply the generic triviality theorem to the projection of the family $\left\{D_{\lambda}^{p}(\delta) \mid \delta>0\right\}$ on the $\delta$-axis. This yields real numbers $d_{0}(\lambda)=0<d_{1}(\lambda)<\cdots<d_{m}(\lambda)<d_{m+1}(\lambda)=\infty$ such that for all $0 \leq i \leq m$ the projection is a trivial fibration above the interval $\left(d_{i}(\lambda), d_{i+1}(\lambda)\right)$.

We now show that we can choose the numbers $d_{i}(\lambda)$ to depend definably on $\lambda$ for $\lambda$ in an interval of the form $\left(0, \lambda_{1}\right)$. Consider the definable set

$$
\mathcal{D}=\left\{\left(\mathbf{x}_{0}, \ldots, \mathbf{x}_{p}, \lambda, \delta\right) \mid\left(\mathbf{x}_{0}, \ldots, \mathbf{x}_{p}\right) \in D_{\lambda}^{p}(\delta), \lambda>0, \delta>0\right\}
$$

and its projection on the $(\lambda, \delta)$-plane. By the generic triviality theorem, the region $\{\lambda>$ $0, \delta>0\}$ can be partitioned in finitely many definable subsets such that the projection of $\mathcal{D}$ is a trivial fibration over each of those subsets. Without loss of generality, we can assume that those subsets are cylindrical cells for the $\lambda$-projection (this is the o-minimal equivalent of cylindrical algebraic decomposition, see Chapter 3 of [8] or Definition 2.4 of [7]). In particular, there exists $\lambda_{1}>0$ and continuous definable functions $d_{0}(\lambda)=$ $0<d_{1}(\lambda)<\cdots<d_{m}(\lambda)<d_{m+1}(\lambda)=\infty$ defined for $\lambda \in\left(0, \lambda_{1}\right)$ such that the cells

$$
C_{i}=\left\{(\lambda, \delta) \mid \lambda \in\left(0, \lambda_{1}\right), d_{i}(\lambda)<\delta<d_{i+1}(\lambda)\right\}
$$

are part of this partition for $0 \leq i \leq m$. Without loss of generality, we can assume that $\lambda_{1} \leq \lambda_{0}$.

The functions $d_{i}(\lambda)$ being definable, each has a well-defined (although possibly infinite) limit when $\lambda$ goes to zero. Let $j$ be the largest index such that $\lim _{\lambda \rightarrow 0} d_{j}(\lambda)=0$, and let $\delta_{0}(\lambda)=d_{j}(\lambda)$ and $\delta_{1}(\lambda)=d_{j+1}(\lambda)$. The functions $\delta_{0}$ and $\delta_{1}$ are definable and verify

$$
\lim _{\lambda \rightarrow 0} \delta_{0}(\lambda)=0 \quad \text { and } \quad \lim _{\lambda \rightarrow 0} \delta_{1}(\lambda)>0 .
$$

Since the projection of $\mathcal{D}$ is a trivial fibration over the cell $C_{j}$, then for any fixed $\lambda \in$ $\left(0, \lambda_{1}\right)$ and any $0 \leq \delta_{0}(\lambda)<\delta^{\prime}<\delta<\delta_{1}(\lambda)$, the inclusion $D_{\lambda}^{p}\left(\delta^{\prime}\right) \hookrightarrow D_{\lambda}^{p}(\delta)$ is certainly a homotopy equivalence.

Let $R>0$ be such that $X_{\lambda} \subseteq\{|\mathbf{x}| \leq R\}$ for all $\lambda \in(0,1)$. We define for $p \in \mathbb{N}$,

$$
\eta_{p}(\lambda)=p(p+1)\left(4 R \max _{\mathbf{x} \in X_{\lambda}}\left|\mathbf{x}-f^{\lambda}(\mathbf{x})\right|+2\left(\max _{\mathbf{x} \in X_{\lambda}}\left|\mathbf{x}-f^{\lambda}(\mathbf{x})\right|\right)^{2}\right) .
$$

By Proposition 15, we have

$$
\lim _{\lambda \rightarrow 0} \eta_{p}(\lambda)=0
$$

Lemma 21. For all $\lambda \in\left(0, \lambda_{0}\right), \delta>0$ and $\varepsilon>0$, the following inclusions hold:

$$
D_{\lambda}^{p}(\delta) \subseteq W_{\lambda}^{p}\left(\delta+\eta_{p}(\lambda)\right) \quad \text { and } \quad W_{\lambda}^{p}(\varepsilon) \subseteq D_{\lambda}^{p}\left(\varepsilon+\eta_{p}(\lambda)\right) .
$$


Proof. Let $m(\lambda)=\max _{\mathbf{x} \in X_{\lambda}}\left|\mathbf{x}-f^{\lambda}(\mathbf{x})\right|$. For any $\mathbf{x}_{i}, \mathbf{x}_{j}$ in $X_{\lambda}$, the triangle inequality gives

$$
\begin{aligned}
\left|f^{\lambda}\left(\mathbf{x}_{i}\right)-f^{\lambda}\left(\mathbf{x}_{j}\right)\right|^{2} & \leq\left[\left|f^{\lambda}\left(\mathbf{x}_{i}\right)-\mathbf{x}_{i}\right|+\left|\mathbf{x}_{i}-\mathbf{x}_{j}\right|+\left|\mathbf{x}_{j}-f^{\lambda}\left(\mathbf{x}_{j}\right)\right|\right]^{2} \\
& \leq\left[\left|\mathbf{x}_{i}-\mathbf{x}_{j}\right|+2 m(\lambda)\right]^{2} \\
& \leq\left|\mathbf{x}_{i}-\mathbf{x}_{j}\right|^{2}+8 R m(\lambda)+4 m(\lambda)^{2}
\end{aligned}
$$

Summing this inequality for all $0 \leq i<j \leq p$, we obtain that for any $\mathbf{x}_{0}, \ldots, \mathbf{x}_{p}$ in $X_{\lambda}$,

$$
\rho_{p}\left(f^{\lambda}\left(\mathbf{x}_{0}\right), \ldots, f^{\lambda}\left(\mathbf{x}_{p}\right)\right) \leq \rho_{p}\left(\mathbf{x}_{0}, \ldots, \mathbf{x}_{p}\right)+\eta_{p}(\lambda) .
$$

The first inclusion follows easily from this inequality. The second inclusion follows from a similar reasoning.

Proposition 22. For any $p \in \mathbb{N}$, there exists $\lambda \in\left(0, \lambda_{0}\right), \varepsilon \in\left(0, \varepsilon_{0}\right)$ and $\delta>0$ such that

$$
H_{*}\left(W_{\lambda}^{p}(\varepsilon)\right) \cong H_{*}\left(D_{\lambda}^{p}(\delta)\right)
$$

Proof. Let $\delta_{0}(\lambda)$ and $\delta_{1}(\lambda)$ be the functions defined in Proposition 20. Since the limit when $\lambda$ goes to zero of $\delta_{1}(\lambda)-\delta_{0}(\lambda)$ is not zero, whereas the limit of $\eta_{p}(\lambda)$ is zero, we can choose $\lambda>0$ such that $\delta_{1}(\lambda)-\delta_{0}(\lambda)>2 \eta_{p}(\lambda)$. Then we can choose $\delta^{\prime}>0$ such that $\delta_{0}(\lambda)<\delta^{\prime}<\delta^{\prime}+2 \eta_{p}(\lambda)<\delta_{1}(\lambda)$. Taking a smaller $\lambda$ if necessary, we can also assume that $\delta^{\prime}+3 \eta_{p}(\lambda)<\varepsilon_{0}$.

Let $\varepsilon=\delta^{\prime}+\eta_{p}(\lambda), \delta=\delta^{\prime}+2 \eta_{p}(\lambda)$ and $\varepsilon^{\prime}=\delta^{\prime}+3 \eta_{p}(\lambda)$. From Lemma 21 we have the following sequence of inclusions:

$$
D_{\lambda}^{p}\left(\delta^{\prime}\right) \stackrel{i}{\hookrightarrow} W_{\lambda}^{p}(\varepsilon) \stackrel{j}{\hookrightarrow} D_{\lambda}^{p}(\delta) \stackrel{k}{\hookrightarrow} W_{\lambda}^{p}\left(\varepsilon^{\prime}\right)
$$

Since both $\varepsilon$ and $\varepsilon^{\prime}$ are less than $\varepsilon_{0}$, Proposition 18 ensures that the inclusion map $k \circ j$ is a homotopy equivalence. Similarly, since both $\delta$ and $\delta^{\prime}$ are in the interval $\left(\delta_{0}(\lambda), \delta_{1}(\lambda)\right)$, it follows from Proposition 20 that the inclusion $j \circ i$ is a homotopy equivalence too. In particular, both inclusions give rise to isomorphisms on the homology level. The resulting diagram in homology is the following:

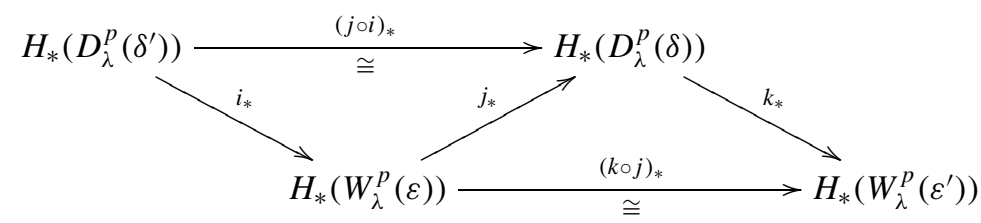

Since $(j \circ i)_{*}=j_{*} \circ i_{*}$, the surjectivity of $(j \circ i)_{*}$ implies that $j_{*}$ must be surjective, and, similarly, the fact that $(k \circ j)_{*}=k_{*} \circ j_{*}$ is injective implies that $j_{*}$ is injective. Hence, $j_{*}$ is an isomorphism between $H_{*}\left(W_{\lambda}(\varepsilon)\right)$ and $H_{*}\left(D_{\lambda}(\delta)\right)$, as required. 
Proof of Theorem 1. The proof of the main theorem now follows easily from the results in this section. If $L$ is the Hausdorff limit of a sequence of fibers $A_{a_{i}}$, we can construct a family $X$ as in Proposition 6 such that $X_{0}=L$. Then, for all $\lambda \in\left(0, \lambda_{0}\right)$, we have

$$
b_{k}(L) \leq \sum_{p+q=k} b_{q}\left(W_{\lambda}^{p}\right)
$$

From Proposition 22, for $\lambda$ small enough, we can find $\varepsilon \in\left(0, \varepsilon_{0}\right)$ and $\delta>0$ such that $b_{q}\left(W_{\lambda}^{p}\right)=b_{q}\left(W_{\lambda}^{p}(\varepsilon)\right)=b_{q}\left(D_{\lambda}^{p}(\delta)\right)$ for every integer $0 \leq p \leq k$. Thus, if $a \in A^{\prime}$ is such that $X_{\lambda}=A_{a}$, inequality (3) in Theorem 1 holds for that $a$.

Remark 23. Note that the upper-bound in Theorem 1 depends only on the sets $D_{a}^{p}(\delta)$, which are defined from the original family $A$ but independent of the auxiliary family $X$. Thus, we will be able to derive quantitative estimates from our main result without ever having to worry about the complexity of the fibers $X_{\lambda}$.

This is an important remark, because the complexity of the description of $X_{\lambda}$ may be much worse than the complexity of a fiber $A_{a}$, even for a choice of $a$ such that the two sets are equal.

\section{Effective Estimates on the Betti Numbers}

This section is devoted to the quantitative estimates than can be derived from Theorem 1, both in the algebraic and the Pfaffian case.

\subsection{Pfaffian Functions}

We start by recalling the basic results about Pfaffian functions. Let $\mathcal{U} \subseteq \mathbb{R}^{n}$ be an open domain.

Definition 24. Let $\mathbf{x}=\left(x_{1}, \ldots, x_{n}\right)$ and let $\left(f_{1}(\mathbf{x}), \ldots, f_{\ell}(\mathbf{x})\right)$ be a sequence of analytic functions in $\mathcal{U}$. This sequence is called a Pfaffian chain if the functions $f_{i}$ are solution on $\mathcal{U}$ of a triangular differential system of the form

$$
d f_{i}(\mathbf{x})=\sum_{j=1}^{n} P_{i, j}\left(\mathbf{x}, f_{1}(\mathbf{x}), \ldots, f_{i}(\mathbf{x})\right) d x_{j},
$$

where the functions $P_{i, j}$ are polynomials in $\mathbf{x}, f_{1}, \ldots, f_{i}$.

If $\left(f_{1}, \ldots, f_{\ell}\right)$ is a fixed Pfaffian chain on a domain $\mathcal{U}$, the function $q$ is a Pfaffian function in the chain $\left(f_{1}, \ldots, f_{\ell}\right)$ if there exists a polynomial $Q$ such that for all $\mathbf{x} \in \mathcal{U}$,

$$
q(\mathbf{x})=Q\left(\mathbf{x}, f_{1}(\mathbf{x}), \ldots, f_{\ell}(\mathbf{x})\right) .
$$

Pfaffian functions come naturally with a notion of complexity, or format. If $\left(f_{1}, \ldots, f_{\ell}\right)$ is a Pfaffian chain, we call $\ell$ its length, and we let its degree $\alpha$ be the maximum of the 
degrees of the polynomials $P_{i, j}$ appearing in (25). If $q$ is as in (26), the degree $\beta$ of the polynomial $Q$ is called the degree of $q$ in the chain $\left(f_{1}, \ldots, f_{\ell}\right)$.

Definition 25. For $q$ as above, the tuple $(n, \ell, \alpha, \beta)$ is called the format of $q$.

Example 26. Let $\mathbf{x}=\left(x_{1}, \ldots, x_{n}\right) \in \mathbb{R}^{n}$ and let $\mathbf{m}_{1}, \ldots, \mathbf{m}_{\ell}$ be fixed vectors in $\mathbb{R}^{n}$. Define for all $1 \leq i \leq \ell, f_{i}(\mathbf{x})=e^{\left\langle\mathbf{m}_{i}, \mathbf{x}\right\rangle}$ (where $\langle\cdot, \cdot\rangle$ denotes the Euclidean scalar product). Then $\left(f_{1}, \ldots, f_{\ell}\right)$ is a Pfaffian chain of length $\ell$ and degree $\alpha=1$ on $\mathbb{R}^{n}$.

The class of Pfaffian functions is a very large class that contains, among other things, all real elementary functions (for a suitable choice of the domain of definition), Liouville functions, and Abelian integrals. We refer the reader to the book [18] or papers [12] and [13] for more details and examples. The main result about Pfaffian functions is the following.

Theorem 27 [18]. Let $\left(f_{1}, \ldots, f_{\ell}\right)$ be a Pfaffian chain of length $\ell$ and degree $\alpha$ defined on $\mathbb{R}^{n}$. Let $\left(q_{1}, \ldots, q_{n}\right)$ be Pfaffian functions in that chain, and let $\left(n, \ell, \alpha, \beta_{i}\right)$ be the format of $q_{i}$ for $1 \leq i \leq n$. Then the number of solutions of the system

$$
q_{1}(\mathbf{x})=\cdots=q_{n}(\mathbf{x})=0
$$

that are isolated in $\mathbb{C}^{n}$ is bounded from above by

$$
2^{\ell(\ell-1) / 2} \beta_{1} \cdots \beta_{n}\left(\beta_{1}+\cdots+\beta_{n}-n+\min (n, \ell) \alpha+1\right)^{\ell} .
$$

Remark 28. In particular, using Example 26 through a logarithmic change of variables, one can show that if $\left(q_{1}, \ldots, q_{n}\right)$ are sparse real polynomials, the number of isolated roots of the system $q_{1}(\mathbf{x})=\cdots=q_{n}(\mathbf{x})=0$ in the quadrant $\left(\mathbb{R}_{+}\right)^{n}$ can be bounded independently of the degrees of the polynomials $q_{i}$. If no more than $\ell$ monomials appear with a non-zero coefficient in at least one of the polynomials, Theorem 27 gives that the number of roots of the system is bounded by $2^{\ell(\ell-1) / 2}(n+1)^{\ell}$. (See [18]. Note that this bound is known to be pessimistic, at least in some cases, see [19] for instance.)

Theorem 27 can be easily generalized to the case where the Pfaffian chain $\left(f_{1}, \ldots, f_{\ell}\right)$ is defined on a domain $\mathcal{U}$ different from $\mathbb{R}^{n}$. In that case the bound (28) becomes

$$
2^{\ell(\ell-1) / 2} \beta^{n} O(n(\alpha+\beta))^{\ell} .
$$

The constant coming from the $O(\cdots)$ notation depends only on the geometry of the open domain $\mathcal{U}$. In many cases (for instance if the functions $q_{i}$ are real elementary functions) the domain $\mathcal{U}$ is given by

$$
\mathcal{U}=\left\{\mathbf{x} \mid g_{1}(\mathbf{x})>0, \ldots, g_{r}(\mathbf{x})>0\right\},
$$

where the functions $g_{i}$ are Pfaffian. In that case a suitable constant can be determined explicitly (see for instance Chapter 1 in [29] for a discussion of the determination of such constants). 


\subsection{Quantifier-Free Formulas}

In this section we let $\mathcal{P}=\left\{p_{1}, \ldots, p_{s}\right\}$ be either a set of polynomials or a set of Pfaffian functions defined in a common Pfaffian chain $\left(f_{1}, \ldots, f_{\ell}\right)$ on a definable domain $\mathcal{U}$. By a quantifier-free formula on $\mathcal{P}$, we mean a Boolean combination of sign conditions on the functions in $\mathcal{P}$, as defined below.

Definition 29. A formula $\Phi$ is called a quantifier-free formula on $\mathcal{P}$ if it is derived from atoms of the form $p_{i} \star 0$, where $1 \leq i \leq s$ and $\star \in\{=, \leq, \geq\}$, using conjunctions, disjunctions and negations. Moreover, we say that the formula $\Phi$ is $\mathcal{P}$-closed if it was derived without using negations.

We endow quantifier-free formulas with the following format. ${ }^{1}$

Definition 30. Let $\Phi$ be a quantifier-free formula on $\mathcal{P}$, where $\mathcal{P}$ is a collection of $s$ polynomials in $n$ variables of degree bounded by $d$. Then $(n, d, s)$ is called the format of $\Phi$.

Similarly, if $\left(f_{1}, \ldots, f_{\ell}\right)$ is a fixed Pfaffian chain and $\mathcal{P}$ is a collection of $s$ functions in that chain, where the format of those functions is at most $(n, \ell, \alpha, \beta)$, then the format of any quantifier-free formula on $\mathcal{P}$ is $(n, \ell, \alpha, \beta, s)$.

If $\Phi$ is a quantifier-free formula on $\mathcal{P}$, where $\mathcal{P}$ is a collection of polynomials, the associated semialgebraic set is $X=\left\{\mathbf{x} \in \mathbb{R}^{n} \mid \Phi(\mathbf{x})\right\}$. If $\mathcal{P}$ is a collection of Pfaffian functions in a Pfaffian chain defined on a domain $\mathcal{U}$ and $\Phi$ is a quantifier-free formula on $\mathcal{P}$, the associated semi-Pfaffian set is the set $X=\{\mathbf{x} \in \mathcal{U} \mid \Phi(\mathbf{x})\}$.

Both polynomials and Pfaffian functions generate o-minimal structures. For polynomials, this is a consequence of the famous Tarski-Seidenberg theorem (see [3] or [8]). The o-minimality of the structure $\mathcal{S}_{\text {Pfaff }}$ generated by Pfaffian functions was first proved by Wilkie [26], and generalized in [17], [20], and [25]. Gabrielov introduced the notion of relative closure in [11] to offer a description of $\mathcal{S}_{\text {Pfaff }}$ more adapted to solving quantitative questions in that structure, and Corollary 3 is an example of how this construction can yield answers in the case of topological complexity (see also [16] for estimates on the number of connected components). The relation between relative closures and Hausdorff limits is discussed in more detail in the Introduction. The reader can also refer to [11], [13], and [16] for a precise description of the construction of $\mathcal{S}_{\text {Pfaff }}$ via relative closures.

\subsection{Betti Number Bounds for Quantifier-Free Formulas}

Elementary Morse theory used in conjunction with the Bézout inequality lets us bound the Betti numbers of real algebraic varieties in terms of the degrees of the defining polynomials. This was first noticed by Oleinik and Petrovsky, and then independently by Thom and Milnor (the reader can refer to Chapitre 11 of [3] for a proof and the

\footnotetext{
${ }^{1}$ Note that this may not be the most standard notion of format for quantifier-free formulas, but it is well-adapted to the Betti number bounds we discuss next.
} 
corresponding references). Khovanskil noted that the method could be generalized to zero-sets of Pfaffian functions [18], [28].

The notion of $\mathcal{P}$-closed formulas was introduced by Basu in the algebraic setting. Using basic algebraic topology techniques and the bound of Oleinik, Petrovsky, Milnor and Thom, he was able to prove the following estimate. ${ }^{2}$

Theorem 31 [1]. Let $\Phi$ be a $\mathcal{P}$-closed polynomial formula of format $(n, d, s)$ and let $X$ be the corresponding semialgebraic set. The sum of Betti numbers of $X$ admits the upper-bound

$$
b(X) \leq O(s d)^{n}
$$

Basu's technique goes through in the Pfaffian setting, and one obtains the following result.

Theorem 32 [28]. Let $\left(f_{1}, \ldots, f_{\ell}\right)$ be a Pfaffian chain on a definable domain $\mathcal{U}$. Let $\mathcal{P}$ be a collection of Pfaffian functions in that chain, and let $\Phi$ be a $\mathcal{P}$-closed formula of format $(n, \ell, \alpha, \beta, s)$. If $X$ is the corresponding semi-Pfaffian set $X=\{\mathbf{x} \in \mathcal{U} \mid \Phi(\mathbf{x})\}$, the sum of the Betti numbers of $X$ admits the following upper-bound:

$$
b(X) \leq 2^{\ell(\ell-1) / 2} s^{n} O(n(\alpha+\beta))^{n+\ell},
$$

where the constant depends only on the definable domain $\mathcal{U}$.

Note that the above bound requires that the constant depends on $\mathcal{U}$ because Khovanskiı̌'s theorem applied on a general domain $\mathcal{U}$ gives a bound of the form (29), that involves a constant depending on the geometry of $\mathcal{U}$.

Remark 33. For any estimate on the Betti numbers of semi-Pfaffian sets, we must assume that the domain $\mathcal{U}$ under consideration is definable. Indeed, one can easily construct non-definable domains $\mathcal{U}$ for which there exists semi-Pfaffian sets $X$ such that $b(X)$ is infinite.

In the case where $X$ is compact, but is not given by a $\mathcal{P}$-closed formula (for instance, if $X$ is a semialgebraic set that has been obtained by a quantifier elimination procedure), bounds on the Betti numbers can be established using the fact that the singular homology of $X$ and its Borel-Moore homology coincide when $X$ is compact. Using the subadditivity of the Borel-Moore homology (see for instance [23, Proposition 1.8], [27], or [5]) along with Theorem 31 and the estimates on the number of sign cells in [2], one can show that the rank of the Borel-Moore groups of a compact semialgebraic set defined by a formula of format $(n, d, s)$ is bounded by $O(s d)^{2 n}$. The same method yields an estimate for semi-Pfaffian sets too [29, Theorem 2.23]. However, these results have been superseded by Theorem 34 below.

\footnotetext{
${ }^{2}$ Basu's estimate is actually slightly sharper than the one given here, but the simpler form (30) is enough for our purposes.
} 
Recently, Gabrielov and Vorobjov used Theorem 31 to establish a Betti number bound valid for any semialgebraic set $X$, with no assumptions either on the topology of $X$ or on the shape of its defining formula. The result they obtained is the following.

Theorem 34 [14]. Let $\Phi$ be a polynomial quantifier-free formula of format $(n, d, s)$ and let $X$ be the corresponding semialgebraic set. The sum of Betti numbers of $X$ admits the following upper-bound:

$$
b(X) \leq O\left(s^{2} d\right)^{n}
$$

Again, the result can be generalized without problem to the Pfaffian case, to obtain the following estimate.

Theorem 35. Let $X$ be any semi-Pfaffian set defined by a quantifier-free formula of format $(n, \ell, \alpha, \beta, s)$. The sum of the Betti numbers of $X$ admits a bound of the form

$$
b(X) \leq 2^{\ell(\ell-1) / 2} s^{2 n} O(n(\alpha+\beta))^{n+\ell},
$$

where the constant depends only on the definable domain $\mathcal{U}$.

\subsection{Proof of Corollaries 2 and 3}

Let $A \subseteq \mathbb{R}^{n} \times \mathbb{R}^{r}$ be a definable family of compact, uniformly bounded sets defined by a quantifier-free formula $\Phi(\mathbf{x}, a)$ whose atoms are sign condition on either polynomials or Pfaffian functions, and let $L$ be a Hausdorff limit of fibers in $A$. According to Theorem 1, we can bound $b_{k}(L)$ by estimating $b\left(D_{a}^{p}(\delta)\right)$ for all $0 \leq p \leq k$ and suitable values $a=a^{*}$ and $\delta=\delta^{*}$ (the precise values of $a^{*}$ and $\delta^{*}$ do not affect the estimate).

Suppose first that the atoms of $\Phi(\mathbf{x}, a)$ are polynomials. Then for any $p, \delta, a$, the set $D_{a}^{p}(\delta)$ is given by the quantifier-free formula $\Psi_{p}\left(\mathbf{x}_{0}, \ldots, \mathbf{x}_{p}, a\right)$ such that

$$
\Psi_{p}\left(\mathbf{x}_{0}, \ldots, \mathbf{x}_{p}, a\right)=\Phi\left(\mathbf{x}_{0}, a\right) \wedge \cdots \wedge \Phi\left(\mathbf{x}_{p}, a\right) \wedge \rho_{p}\left(\mathbf{x}_{0}, \ldots, \mathbf{x}_{p}\right) \leq \delta .
$$

The set of functions $\mathcal{Q}_{p}$ appearing in the atoms of $\Psi_{p}$ are of the form $f\left(\mathbf{x}_{i}, a\right)$, for any $0 \leq i \leq p$ and any $f \in \mathcal{P}$, plus of course $\rho_{p}\left(\mathbf{x}_{0}, \ldots, \mathbf{x}_{p}\right)-\delta$. Thus, specializing $\Psi_{p}$ at $a=a^{*}$ gives a formula of format $(n(p+1), d, s(p+1)+1)$, where $s=\mathcal{P}$ and

$$
d=\max \left(2,\left\{\operatorname{deg}_{\mathbf{x}} f(\mathbf{x}, a) \mid f \in \mathcal{P}\right\}\right) .
$$

Theorem 34 gives $b_{q}\left(D_{a}^{p}(\delta)\right) \leq O\left(p^{2} s^{2} d\right)^{(p+1) n}$, and Corollary 2 follows.

The same reasoning goes through in the Pfaffian case. If after specialization the format of $\Phi\left(\mathbf{x}, a^{*}\right)$ is $(n, \ell, \alpha, \beta, s)$, then the format of $\Psi_{p}\left(\mathbf{x}_{0}, \ldots, \mathbf{x}_{p}, a^{*}\right)$ is $(n(p+1)$, $\ell(p+1), \alpha, \beta, s(p+1)+1) .{ }^{3}$ From Theorem 35, we obtain that

$$
b\left(D_{a}^{p}(\delta)\right) \leq 2^{\ell(p+1)[\ell(p+1)-1] / 2} s^{2 n(p+1)} O(n p(\alpha+\beta))^{(p+1)(n+\ell)},
$$

\footnotetext{
${ }^{3}$ The length of the Pfaffian chain is multiplied by $p+1$ since we need a copy of each function in the chain for every one of the blocks of variables $\mathbf{x}_{0}, \ldots, \mathbf{x}_{p}$.
} 
and thus (4) holds for $L$. This is true for any number of parameters $r$ in the family $A$, and Corollary 3 is simply the special case where $r=1$.

Remark 36. If the fiber $A_{a}$ is defined by a $\mathcal{P}$-closed formula, then the formula $\Psi_{p}$ defining $D_{\lambda}^{p}(\delta)$ is a $\mathcal{Q}_{p}$-closed formula, and the dependence on $s$ of the bound on $b_{k}(L)$ can be improved from $s^{2 n(k+1)}$ to $s^{n(k+1)}$ by using the tighter estimates available in that case (Theorem 31 in the algebraic case and Theorem 32 in the Pfaffian case).

\section{Acknowledgments}

I am grateful to my advisors Andrei Gabrielov and Marie-Françoise Roy for their help and support. I also thank Saugata Basu, Michel Coste, Jean-Marie Lion and Nicolai Vorobjov for helpful discussions, and the anonymous referees for their careful reading and their many pertinent suggestions.

\section{References}

1. S. Basu. On bounding the Betti numbers and computing the Euler characteristic of semi-algebraic sets. Discrete Comput. Geom., 22 (1999), 1-18.

2. S. Basu, R. Pollack, and M.-F. Roy. On the number of cells defined by a family of polynomials on a variety. Mathematika, 43 (1996), 120-126.

3. J. Bochnak, M. Coste, and M.-F. Roy. Géométrie algébrique réelle. Springer-Verlag, Berlin, 1987. Second edition in English: Springer-Verlag, Berlin, 1998

4. L. Bröcker. Families of semialgebraic sets and limits. In Real Algebraic Geometry (Rennes, 1991), pp. 145162. Lecture Notes in Mathematics, Vol. 1524. Springer-Verlag, Berlin, 1992.

5. P. Bürgisser. Lower bounds and real algebraic geometry. In Algorithmic and Quantitative Real Algebraic Geometry, pp. 35-54. DIMACS: Series in Discrete Mathematics and Theoretical Computer Science, Vol. 60. American Mathematical Society, Providence, RI, 2003.

6. M. Coste. Topological types of fewnomials. In Singularities Symposium-Lojasiewicz 70 (Kraków, 1996; Warsaw, 1996), pp. 81-92. Banach Center Publications, Vol. 44. Polish Academy of Science, Warsaw, 1998.

7. M. Coste. An Introduction to O-minimal Geometry. Dip. Mat. Univ. Pisa, Dottorato di Ricerca in Matematica, Istituti Editoriali e Poligrafici Internazionali, 2000. Also available on http: //www. ihp-raag.org/.

8. L. van den Dries. Tame Topology and O-Minimal Structures. LMS Lecture Note Series No. 248. Cambridge University Press, Cambridge, 1998

9. L. van den Dries. Hausdorff limits under tame conditions. In preparation.

10. S. Eilenberg and N. Steenrod Foundations of Algebraic Topology. Princeton University Press, Princeton, NJ, 1952.

11. A. Gabrielov. Relative closure and the complexity of Pfaffian elimination. In Discrete and Computational Geometry: The Goodman-Pollack Festschrift, pp. 441-460. Algorithms and Combinatorics, Vol. 25. Springer-Verlag, Berlin, 2003.

12. A. Gabrielov and N. Vorobjov. Complexity of stratifications of semi-Pfaffian sets. Discrete Comput. Geom., 14 (1995), 71-91.

13. A. Gabrielov and N. Vorobjov. Complexity of computations with Pfaffian and Noetherian functions. To appear in Normal Forms, Bifurcations and Finiteness Problems in Differential Equations, edited by Y. Ilyashenko and C. Rousseau. NATO Science Series II, Vol. 137. Kluwer Academic, Dordrecht, 2004.

14. A. Gabrielov and N. Vorobjov. Betti numbers for quantifier-free formulae. To appear in Discrete Comput. Geom. Available at http://www. math.purdue.edu/ agabriel/preprint.html. 
15. A. Gabrielov, N. Vorobjov, and T. Zell. Betti numbers of semialgebraic and sub-Pfaffian sets. J. London Math. Soc., 69 (2004), 27-43.

16. A. Gabrielov and T. Zell. On the number of connected components of the relative closure of a semi-Pfaffian family. In Algorithmic and Quantitative Real Algebraic Geometry, pp. 65-75. DIMACS: Series in Discrete Mathematics and Theoretical Computer Science, Vol. 60. American Mathematical Society, Providence, RI, 2003.

17. M. Karpinski and A. Macintyre. A generalization of Wilkie's theorem of the complement, and an application to Pfaffian closure. Selecta Math. (N.S.), 5 (1999), 507-516.

18. A. G. Khovanskiǔ. Fewnomials. American Mathematical Society, Providence, RI, 1991.

19. T.-Y. Li, J. M. Rojas, and X. Wang. Counting real connected components of trinomials curve intersections and $m$-nomial hypersurfaces. Discrete Comput. Geom., 30 (2003), 379-414.

20. J.-M. Lion and J.-P. Rolin. Volumes, Feuilles de Rolle de Feuilletages analytiques et Théorème de Wilkie, Ann. Fac. Sci. Toulouse Math., 7 (1998), 93-112.

21. J.-M. Lion and P. Speissegger. A geometric proof of the definability of Hausdorff limits. RAAG Preprint 31, available at http://www. ihp-raag.org/. To appear in Selecta Math.

22. D. Marker and C. Steinhorn. Definable types in o-minimal theories. J. Symbolic Logic, 59 (1994), 185-198.

23. J. L. Montaña, J. E. Morais, and L. M. Pardo. Lower bounds for arithmetic networks, II: Sum of Betti numbers. Appl. Algebra Engrg. Comm. Comput., 7 (1996), 41-51.

24. A. Pillay. Definability of types, and pairs of O-minimal structures. J. Symbolic Logic, $\mathbf{5 9}$ (1994), 1400-1409.

25. P. Speissegger. The Pfaffian closure of an o-minimal structure. J. Reine Angew. Math., 508 (1999), 189-211.

26. A. J. Wilkie. A theorem of the complement and some new o-minimal structures. Selecta Math. (N.S.), 5 (1999), 397-421.

27. A. Yao. Decision tree complexity and Betti numbers. J. Comput. System Sci., 55 (1997), 36-43.

28. T. Zell. Betti numbers of semi-Pfaffian sets. J. Pure Appl. Algebra, 139 (1999), 323-338.

29. T. Zell. Quantitative study of semi-Pfaffian sets. Ph.D. thesis, 2003. Available at arxiv.org/abs/ math.AG/0401079.

Received July 28, 2003, and in revised form March 23, 2004. Online publication October 20, 2004. 